\title{
Smart models for a new participatory and sustainable form of governance
}

\author{
G. Perillo \\ Department of Technology, Naples Parthenope University, Italy
}

\begin{abstract}
A region may be defined as smart when it focuses its efforts on the development of human and social capital, on transportation, on information and communication technologies (ICT), on an appropriate management of natural resources and on the promotion of participatory governance. The smart city model calls for the development of medium and small communities in order to provide an answer and a real alternative to the progressive loss of quality of life that is taking place in today's cities.

It is a response to the many problems associated with globalization, which now more than ever seem to deny opportunities for development rather than foster them and loses sight of the centrality of the human being and citizen. Ultimately, it is a model of territorial intelligence that begins with an analysis of strengths and weaknesses and then implements a series of strategic interventions in order to overcome any critical factors and to improve the quality of city life.

Starting from the assumption that we are living in a historic period of great and rapid transformation in which significant changes that result in global economic and geopolitical restructuring are leading us into a new world with new needs, this paper aims to highlight those features that place a particular emphasis on smart development:

- the development of better integrated and more inclusive urban models, by rethinking the city, its logic and its traditional structures;

- the co-partnership of all institutions and all citizens, through information and communication;

- the strategic management of natural resources;

- the creation of new patterns of mobility.
\end{abstract}

Innovation underpins any response to these new needs and it is crucial to the rapid achievement of targets. It is the essential element for interconnecting all physical systems through digital technologies, but it should be borne in mind that 
creating a smart city cannot be a merely technological challenge but it must, above all, be a social one.

\section{Reports on renewable energy sources}

The European Commission's communication of 6 June 2012 on "Renewable Energy: a major player in the European energy market” laid down guidelines for the coming years. The Commission recalls the previous Energy Roadmap 2050 and warns of the need for "early policy clarity on the post 2020 regime" in order to "generate real benefits for investors in industry and infrastructure as well as for renewable energy investors directly" by integrating renewable energies into the single market and opening up the electrical energy market; this will require a serious and profound transformation of the infrastructure, a new awareness on the part of consumers and the promotion of technological innovation and sustainability [1-3].

The International Renewable Energy Agency (IRENA) stresses that energy efficiency, energy saving and above all renewable energy sources represent the future of the world economy but it also focuses on one of the main problems of uncoordinated and incoherent energy policies, namely that policy makers have not yet grasped their full importance. This may be partly due to the fact that they are often unaware of the most recent developments regarding the costs, technologies and other aspects of renewable energy sources [4].

Following a report on the European legislative puzzle regarding incentive schemes, the Council of European Energy Regulators (CEER), a non-profit organisation set up by the national energy market regulators, underlines the need for further, thorough investigations but highlights the benefits that would result from a medium to long-term intervention to harmonise the relevant legislations of the various member states:

- a reduction in the costs needed to reach objectives;

- economies of scale;

- a larger and more dynamic certificates market;

- a more efficient development of renewable energies in areas where there is a higher density of resources.

Such a harmonisation would, of course, not mean uniform sterility but political and legislative compatibility and sustainability [4].

To sum up, these reports point out:

- that many potentially "attractive" areas for the development of the renewable energy industry must tackle a number of issues, such as inadequate networks, the lack of coherent planning at regional and central levels, and uncertainty regarding legislation and authorisation processes. These constitute an obstacle to reaching environmental objectives while assuring the socio-economic growth that stems from the introduction of innovative technologies into the market (6); 
- the need for "energy policy to envisage environmental sustainability objectives";

- that the transition towards an environmentally sustainable energy system could provide a formidable opportunity for the overall growth of the economy;

- the importance of empowering consumers "so as to align the reaching of legitimate individual goals with the interests of the overall system and in particular with those of consumers";

- the rigour versus growth balance cannot be established simply and at random. It must be determined according to the specific context typifying each of the regulated sectors and taking into account the significant changes in progress.

The common denominator is cultural sustainability: the indispensable prerequisite to conceiving and then achieving a broadly sustainable future [5].

In particular, with reference to the this type of sustainability, the most important investment that can be made is "the immaterial one aiming to enhance the technical credibility and accountability of decisions by consolidating the notice and comment procedures, that is to say through consultation and participation in regulatory choices".

Ultimately this notice and comment must overcome its primary limitation, namely that it is aimed at those working in the regulated sectors, so that it can be understood by the general public. It can then be employed to achieve the previously mentioned empowerment and hence ensure a more aware participation in energy matters and break away from the countless instruments of mass distraction that have so far been used [5, 6].

Finally, the aim of the Clean Energy Trends report drafted by Clean Edge Inc. is to monitor the five main trends in the renewable energy sector in order to perform a pioneering function and offer increasingly precise responses. These five trends constitute the already sufficiently analytical core of an energy program worthy of that name, also and above all because its proactive and integrated nature mean that it has been conceived with the aim of planning a sustainable future today [6]:

- the military field;

- the effects of the 2011 Japanese tsunami on world energy policy;

- the retrofit operations to upgrade energy efficiency;

- the contextualised waste-to-energy sector;

- technologies for storing renewable energy.

\section{Smart models}

\subsection{Smart cities}

A region may be defined as smart when it focuses its efforts on the development of human and social capital, on transportation, on information and communication technologies (ICT), on an appropriate management of natural resources and on the promotion of participatory governance. The smart city 
model calls for the development of medium and small communities in order to provide an answer and a real alternative to the progressive loss of quality of life that is taking place in today's cities.

It is a response to the many problems associated with globalization, which now more than ever seems to deny opportunities for development rather than foster them and loses sight of the centrality of the human being and citizen. Ultimately, it is a model of territorial intelligence that begins with an analysis of strengths and weaknesses and then carries out a series of strategic interventions in order to overcome any critical factors and to improve the quality of city life [5-7].

The initial assumption is that we are living in a historic period of great and rapid transformation in which significant changes that result in global economic and geopolitical restructuring are leading us into a new world with new needs, the most important of which concern (fig. 1):

- the development of better integrated and more inclusive urban models, by rethinking the city, its logic and its traditional structures;

- the co-partnership of all institutions and all citizens, and the importance of information and communication;

- the strategic management of natural resources;

- the creation of new patterns of mobility.

Underpinning these needs is innovation: "a factor crucial to any response to these new needs and to the rapid achievement of targets". Innovation must be seen as an empowering factor that, on the one hand, can bring about the interconnection of all physical systems and digital technologies but, on the other hand, it should be borne in mind that creating a smart city "cannot be a merely technological challenge but it must, above all, be a social one".

The considerable efforts that such a transformation requires will be rewarded with "a recovery of efficiency, available time, productivity and a reduction in transaction costs that translate into additional growth for the country of 8-10 GDP points a year, without counting non-quantifiable returns in terms of international competitiveness and image, social cohesion, creativity, innovation, spread of knowledge and livability”.

\subsection{Smart mobility}

In order to highlight the ambitions envisioned by the idea of smart mobility, we must first clearly specify the extent, the entity and the benefits of the changes it entails, not just in terms of intelligent modernisation and innovation but also, and significantly, in social, cultural and behavioural terms.

Here too it is necessary to start from an informed acceptance of the sheer entity of the current economic transition, the speed of which was somewhat unexpected and is generating an equally rapid change in the paradigms of movement.

"The massive increase in world demand for connectivity and movement will soon reach critical levels and, within the next 20 years, it will exceed the capacity of current transport systems, impacting extensively on productivity, on the environment and on societies themselves. At the same time, this demand will 
radically change in terms of critical needs and requirements: this will call for new, more flexible, more adaptive and more personalised solutions”. It will call for smart mobility enabled by technological progress which will make it possible to intervene on the existing system $[6,7]$, optimising it so as to meet immediate needs and, at the same time, introduce structural modifications to the models of supply and demand in order to accompany the long term transition.

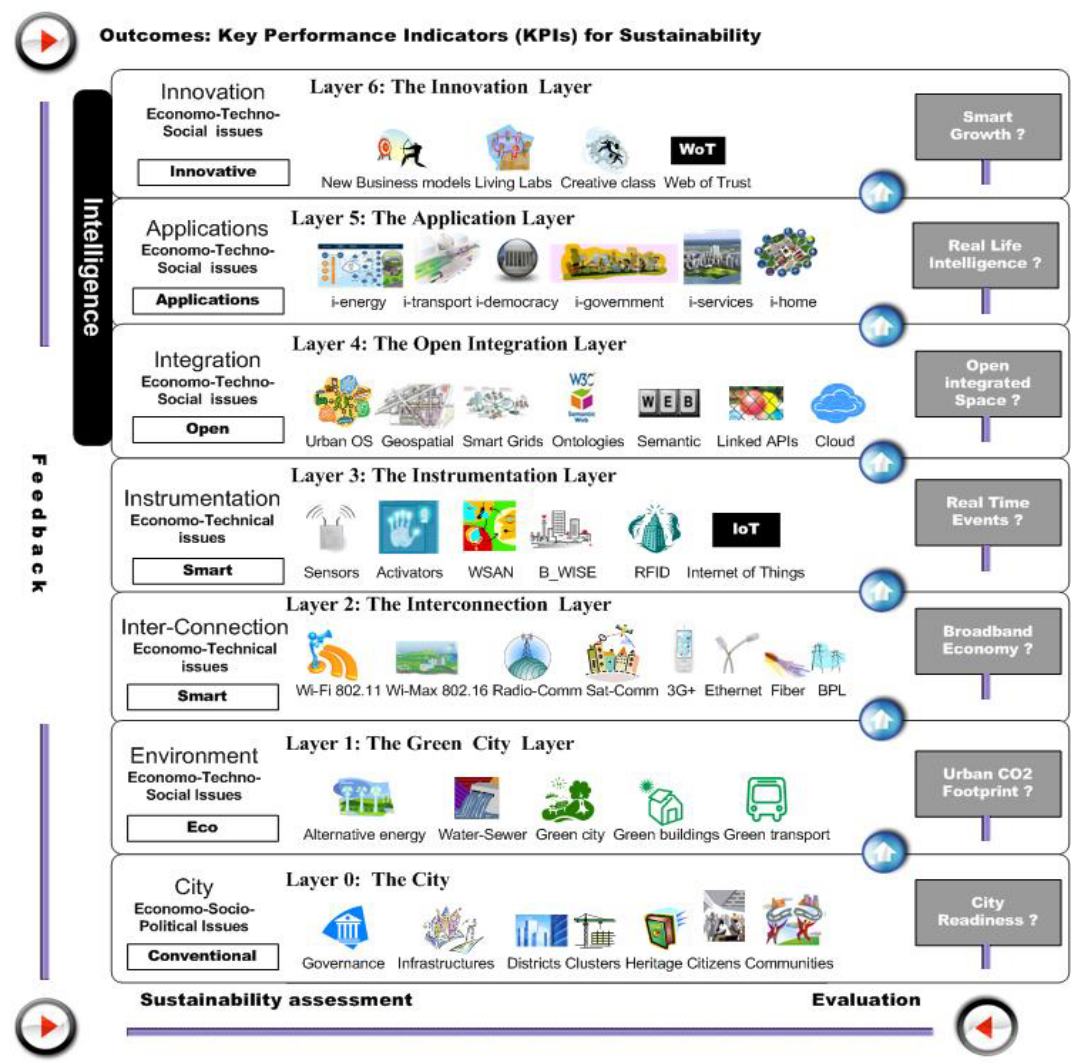

Figure 1: $\quad$ Layers of increasing the sustainability.

Innovation is the fulcrum of all smart options [6-8].

Obviously, we are talking about a technological innovation "which goes beyond the environmental aspects or the creation of new infrastructure: we have to rethink the transport and connectivity systems designed for $20^{\text {th }}$ century needs, making them functional to service the requirements of $21^{\text {st }}$ century industry and society. Facing these new challenges with the tools and approaches of the past would be doomed to failure”.

As a result, technological enablement making mobility smart will have to be able to: 
- manage mobility flows in order to reduce congestion, down times, disservices and risks and also to modify demand for mobility;

- plan mobility systems that can reutilise existing facilities (infrastructural retrofit) in order to obtain "more rational networks that are effective in meeting needs and adapting to emergencies";

- design new infrastructure using a different approach to make it more interactive, more functional and less costly;

- provide citizens with more flexible, useful and personalised services that offer a more effective response to the market's different needs and, at the same time, make them more responsible for their individual behaviours;

- be an empowering factor for smart cities.

The transition to smart mobility - which is becoming a commodity of social and economic life - is inevitable.

Meeting the three key challenges of modern mobility:

- efficiency and efficacy;

- $\quad$ safety of users and system reliability;

- sustainability, accessibility, usability and inclusivity;

calls for the same exchange of ideas as for smart cities. In other words, it is necessary to participate, "to move ideas and move positions" in order to open (and close) the development circle: a virtuous circle to ensure continuous renewal and innovation.

Smart mobility can be worth up to 5 GDP points a year, by enhancing creativity, activating and attracting new investment, increasing safety and reducing social costs.

\section{How to become smart}

It is necessary to generate a high-level science and promote interventions that must be able to "develop technological solutions, services, models and methodologies at the cutting edge of applied industrial and academic research. The application perimeter is provided by Smart Communities, that is to say by the development of innovative models aiming to find the solution to problems on an urban, metropolitan and, more generally, regional scale by means of a set of technologies, applications and models for integration and inclusion”.

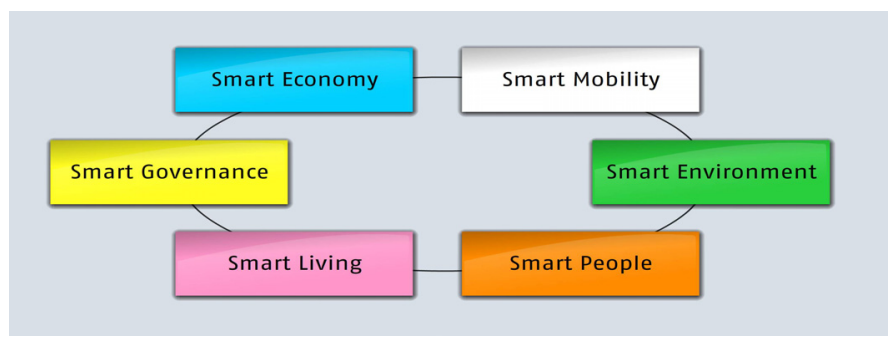

Figure 2: $\quad$ Smart complex models. 
Such interventions must be incorporated into the scheme of the following multi-sustainability (fig. 2):

a) Environmental sustainability

This concept class includes:

- regional safety. The design ideas to be developed in this context must sustain the development of new knowledge, of innovative technologies and of new integrated systems for risk prevention, the defence of the regional environment and related safety measures, by means of ICT solutions which make it possible to step up efficiency in the management of the various systems and in handling emergencies resulting from natural disasters;

- (correct) waste management. The final objective is to contribute to the eco-sustainable development of new management procedures and new technologies to prevent the production of waste upstream and its recycling downstream;

- a new form of overland mobility, achieved through the "promotion of maritime, metropolitan, rail and/or road mobility, the development of technologies and innovative ICT solutions aiming to improve the interoperability of maritime logistic information systems or between maritime, metropolitan, road and/or rail infomobility systems”. Falling within this context is last-mile logistics, the development of which will have to promote new, eco-sustainable models for the logistics sector, partly through the development of systems and technologies that can improve efficiency in the management of goods distribution circuits;

- the improved management of water resources, with a particular focus on the promotion of methods for safeguarding such resources through the development of innovative systems and technologies that will i) improve water management by means of increased efficiency of the network and the existing distribution facilities; ii) monitor, determine and reduce pollutants in water systems; iii) reduce water use in agriculture and industry;

- safeguarding public health, which will also have to be pursued with the aid of new technologies: "functional and empowering ICT solutions and components which make it possible to activate new models of activities in the field of health and wellbeing, the development of e-health services at a national, regional, local and individual level, and improved interaction between health facilities".

b) Social sustainability

This includes:

- ageing communities, stressing the need to improve the quality of life and healthcare of the elderly with the aid of new technologies that can facilitate mobility and thus reduce social isolation; 
- the development of a new system of welfare and a new, inclusive social model that can (re)insert those living on the margins of society (whether for reasons of social status, race or mental health) into everyday city life;

- a new justice system that must be efficient and swift, contributing pro rata to the reduction of costs currently incurred (not only in financial terms but also in respect of time wasted unnecessarily);

- the modernisation of the compulsory education system, in terms both of the tools used (the widespread introduction of ICT) and of teaching methods, through personalised teaching and training courses, flexible timetables, scheduled attendance, a dynamic organisation of classes and tools for student management.

\section{c) Cultural sustainability}

By means of projects carried out in the culture/heritage field, participants will be required to promote the development of "new technological solutions for the diagnostic assessment, restoration, conservation, digitization and enjoyment of material and non-material cultural heritage in order to enhance its impact in environmental, touristic and cultural terms and to facilitate the integration of innovative public and private sector services, also with reference to the region's ability to attract tourists and visitors”.

\section{d) Technological sustainability}

The technologies of the future that must be favoured include:

- home automation, the development of which must foster the promotion of new knowledge, innovative technological solutions and highly innovative systems, constructions and products that "on the basis of an Ambient Intelligence and Ambient Assisted Living framework, make it possible to redesign the domestic environment so as to ensure a better quality of life, social inclusion, safety and also total autonomy for the differently able";

- smart grids;

- sustainable architecture and materials, with the development of new solutions, technologies and high-performance materials which aim, in accordance with the principle of Zero lmpact Building, to improve energy efficiency, lessen environmental impact, monitor and reduce pollution factors, improve health conditions in the home and work environments and ensure greater safety and comfort to users;

- cloud computing technologies for smart government; this will call for the widespread introduction of cloud platforms and their related applications and services in order to "improve the quality and accessibility of services, guarantee high standards of interoperability between different cloud systems, promote reference implementations based on open source solutions and reduce adoption costs for businesses operating in the new ICT sector in order to increase returns on investment and cut the time to market of their products and services”. 


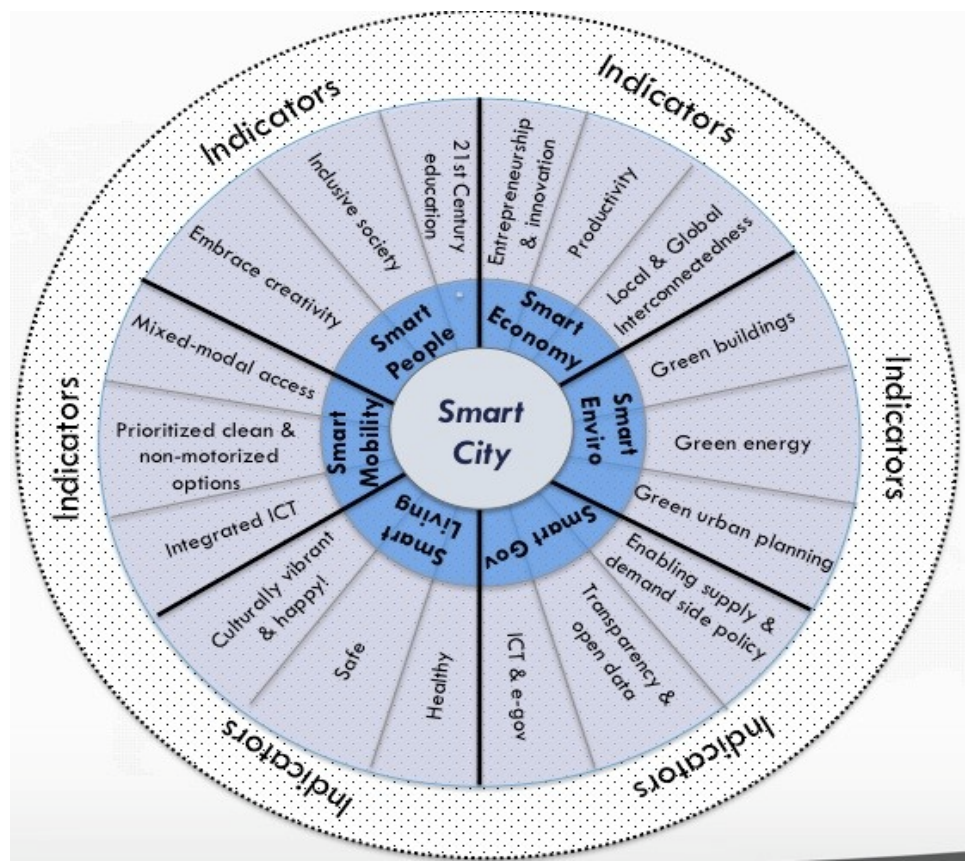

Figure 3: $\quad$ Smart indicators.

\section{Conclusions}

The transformations that interconnected smart models require if we are to create the multi-sustainable society of the future (while benefitting in the mid-term from the increasing advantages generated by their progressive implementation) are undeniably numerous and complex (fig. 3).

However, it must be taken into account that an overall smart model is much more than the mathematical sum of the individual smart features of which it is comprised. Individual sector policies, individual, occasional and potentially selforganised projects, and individual technologies applied within the framework of a highly heterogeneous set of solutions generically labelled as 'smart' "do not make a region truly smart. Technologies, projects and policies must be subordinated to a common goal, otherwise there is a clear risk that the potential of the concept will be diminished and the efforts weakened".

In short, smart models have the potential to offer a win-win scenario as "they minimise the effort required for lesser needs and fully satisfy greater needs, guaranteeing a high quality of life by optimising resources and spaces to assure sustainability". The achievement of this objective calls for the organic implementation of smart models in line with a new cultural spirit that must be integrated, coordinated, scheduled and therefore authoritative. This would put us on a (more) sustainable path (or a less unsustainable one) towards the future. It is a path that, in the future, will hopefully let us make (more) sustainable choices, 
not reluctantly but willingly and as part of a far sighted vision. A sustainable vision.

\section{References}

[1] Berthon B., Guittat P. “Ascesa della città intelligente”, Outlook, n.2, 2011.

[2] Bhardwaj D., Dodgson M., Gann D. M., "Physical-digital integration in city infrastructure”, IBM Journal of Research \& Development, vol. 55, n.1-2, 2011.

[3] Commissione Europea, "Intelligent Transport Systems in Action, action plan and legal framework for the deployment of intelligent transport systems (ITS) in Europe”, 2011.

[4] Commissione Europea, "Smart Cities and Communities - European Innovation Partnership”, 2012.

[5] Dodgson M., Gann D. M., "Technological Innovation and Complex Systems in Cities”, Journal of Urban Technology, vol. 18, n. 3, 2011.

[6] Economist Intelligence Unit, "Liveable cities. Challenges and opportunities for policymakers”, 2010.

[7] Dodgson M., Gann D. M., “Technological Innovation and Complex Systems in Cities”, Journal of Urban Technology, vol. 18, n. 3, 2011.

[8] Economist Intelligence Unit, "Liveable cities. Challenges and opportunities for policymakers”, 2010. 